\title{
Fringing Field Capacitive Smart Sensor Based on PCB Technology for Measuring Water Content in Paper Pulp
}

\author{
Flávio Morais, ${ }^{1}$ Pedro Carvalhaes-Dias $\mathbb{D}^{2,3}$ Luís Duarte ${ }^{\mathbb{D}},{ }^{2}$ Eduardo Costa, ${ }^{3}$ \\ Alexandre Ferreira, ${ }^{3,4}$ and José Siqueira Dias $\mathbb{1 D}^{3}$ \\ ${ }^{1}$ Faculty of Science and Engineering, São Paulo State University Júlio de Mesquita Filho, Tupã, SP, Brazil \\ ${ }^{2}$ Paraná Federal University of Technology-UTFPR, Cornélio Procópio, PR, Brazil \\ ${ }^{3}$ Department of Semiconductors, Instrumentation and Photonics, FEEC/UNICAMP, SP, Brazil \\ ${ }^{4}$ Uninove Campus Vergueiro, São Paulo, SP, Brazil \\ Correspondence should be addressed to José Siqueira Dias; siqueira@demic.fee.unicamp.br
}

Received 20 September 2019; Revised 19 December 2019; Accepted 23 January 2020; Published 4 March 2020

Academic Editor: Alberto J. Palma

Copyright (C) 2020 Flávio Morais et al. This is an open access article distributed under the Creative Commons Attribution License, which permits unrestricted use, distribution, and reproduction in any medium, provided the original work is properly cited.

\begin{abstract}
We present a capacitive smart sensor based on printed circuit board (PCB) technology to measure the amount of water content in a paper pulp at the wet end of a paper machine. The developed sensor incorporates in the same PCB the signal processing circuits. It is a handheld portable device, and its output is sent to the reading equipment using a Bluetooth wireless connection, providing to the sensor's operator ease of mobility around the wet end of a paper machine. The prototype was tested in a laboratory, using a wire mesh to emulate the end of a paper machine, and we were able to measure and easily detect when it reaches the water content in the range of $90 \%$ to $92 \%$, as required in the paper fabrication process. Standard deviation of the capacitance measurements at various moisture levels is four orders of magnitude smaller than the mean. The smart sensor was tested in the $20^{\circ} \mathrm{C}$ to $40^{\circ} \mathrm{C}$ temperature range, in a paper pulp with a gravimetric water content of $91 \%$. Since the variation of capacitance with temperature is practically linear, we propose a simple linear compensation equation to correct the observed sensitivity with the temperature. To keep the signal processing circuits small, low cost, simple, and robust, a novel direct interface sensor to microcontroller circuit technique was used to make the capacitive measurement, allowing for measuring small capacitance deviations without high-frequency oscillators. It was shown that it is possible to integrate the signal processing circuits in the top layer of the PCB interdigitated sensor without adding noise or degrading the performance of the capacitive sensor.
\end{abstract}

\section{Introduction}

In most paper making machines, the properties of the paper are measured only at the dry end of the machine, after the paper is ready. Thus, if the paper that is being produced does not have an acceptable quality, the paper is discarded (probably to be recycled into a paper with lower quality), and the machine's parameters are altered so that the produced paper meets the desired quality standard.

Therefore, being able to measure the water content of the paper pulp at the wet end of a paper machine is fundamental to control the quality of the paper, reduce waste, and minimize the effects in the environment, leading to environmentally friendly manufacturing processes. Many techniques have been proposed to measure the water content in the paper pulp in the wet end of the paper machine, before it enters the fabrication process.

Some techniques are very simple and are capable of only giving a rough estimate of the water content, like the use of fans. In this technique, a conventional fan is rotated inside the paper pulp, and by measuring the motor's torque, it is possible to estimate the water content in the paper pulp [1]. This method is not precise because for very low pulp concentration (high water content in the pulp) the fan rotates freely.

Microwave closed cavity resonators can estimate the water content in the paper pulp with a very high resolution, and fibre concentrations as low as $0.6 \%$ have been measured with a $0.03 \%$ standard deviation [2]. However, the method 


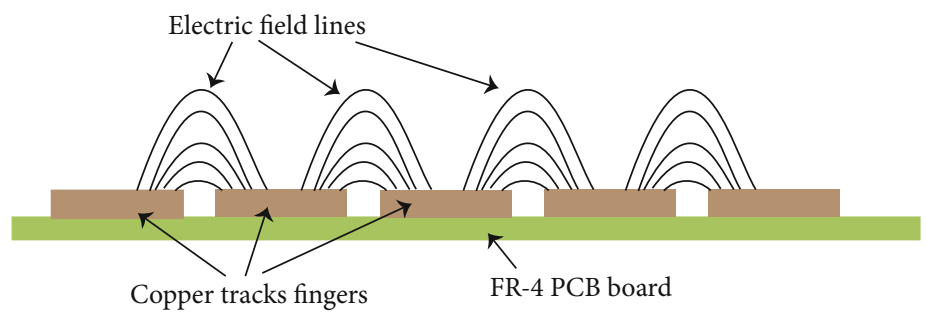

FIGURE 1: Electric flux in the vicinity of the sensor's fingers.

cannot be used in the monitoring of paper pulp in the wet end of the paper machine, since it is necessary to manually take a sample of paper pulp and insert it in the closed cavity resonator. Besides this limitation, it requires very complex instrumentation, since a spectrum analyser and a tracking generator are required.

Other techniques based on microwave attenuation of the material have been proposed [3], but these techniques are difficult to use, specially with low attenuation materials because the attenuation measurements are influenced by multiple reflections.

A microwave open cavity resonator measurement system that would allow usage in the production line has been proposed [4]. However, the resonance frequency of paper pulp is around $2.6 \mathrm{GHz}$. Most cellphone communication systems operate at $2.4 \mathrm{GHz}$, and to avoid interference from the cellphone signals, the microwave sensors must be shielded, so the open cavity sensor is impractical.

An electromagnetic field perturbation sensor was also proposed, to measure the conductivity of the paper pulp, and from these measurements, the water content in the pulp can be estimated [5-8]. However, this technique presents two weaknesses: (a) it is necessary to assume that all the water in the pulp is absorbed by paper fibres, so that the electrical conductivity is due only to the water molecules and (b) different additives can be used in the paper pulp, leading to different conductivities of the material for the same paper pulp with a given water content.

Typically, the paper pulp arrives with high volumetric percentage amount of water content $\left(\theta_{w} \approx 99 \%\right)$ at the wet end of the paper machine and it must be dried until it reaches the $\theta v=90-92 \%$ range before it goes to the next fabrication step. Sundara-Rajan et al. [9] investigated a metal grid fringing field capacitance sensor with an impedance spectroscopy instrumentation to measure moisture content in paper pulp and concluded that from the measured impedance data, the dependence of both admittance and phase on the moisture content is not important (or totally unreliable, as in the case of the measured phase). On the other hand, it was observed that the variation of capacitance for a water pulp content $\theta_{w}$ changing from $90 \%$ to $96 \%$, resulted in a $\Delta C=0.6 \mathrm{pF}$.

Based on the promising results obtained in the study conducted in [9], in this work, we present a wireless capacitive fringing field smart sensor based on a interdigitated printed circuit board (PCB) sensor to measure water content in paper pulp.

\section{The PCB Capacitive Sensor}

2.1. Principle of Operation of Fringing Field Capacitive Sensors. Low-cost fringing fields capacitive sensors using conventional metal grids or PCB fabrication techniques have been successfully presented for many applications, like soils [10], cereal grains [11], air [12, 13], and human skin [14].

A conventional PCB fringing field capacitive sensor is composed of interdigitated coplanar electrodes (usually called fingers), made with the copper tracks of a PCB, as shown in Figure 1. The capacitance of a structure with fingers length $l$, distance between two fingers $d$, thickness of the copper $h$, and $n$ fingers is given by

$$
C \approx \epsilon_{0} \epsilon_{r} \frac{n l h}{d},
$$

where $\epsilon_{0}$ and $\epsilon_{r}$ are, respectively, the permittivity of free space and the relative permittivity of the dielectric material between two fingers. A correction factor in Equation (1) may be necessary if the condition $l h \gg d^{2}$, but with usual values used in these sensors, this is not necessary.

When the material which is in contact with the PCB changes its $\epsilon_{r}$ (for example, the paper pulp in contact with the $\mathrm{PCB}$ ), the sensor's capacitance changes and these variations can be related to the moisture content in the paper pulp [9].

In [10], it has been shown that a small ionic content of the water over the PCB sensor did not alter its response: with the water over the sensor changing its conductivity from $\sigma=32.2 \mathrm{mS} / \mathrm{m}$ to $\sigma=5.7 \mathrm{mS} / \mathrm{m}$, the observed change in the measured capacitance was only $0.02 \%$.

However, depending on the type of paper being produced, a high content of several additives (such as titanium dioxide and alkalis) are added to the paper pulp, and it is recommended to perform a calibration of the sensor in each type of paper pulp. It is very important to notice that the absolute value of the measured capacitance is not important, since we are interested only in which values in the sensor's output indicate that the water content in the paper pulp is within the required range.

\section{The Developed Smart Sensor}

One important characteristic of the capacitive PCB sensors, which has not yet been explored, is the use of this technique to the fabricate smart sensors, since all the signal processing 
circuits could, in principle, be fabricated on the top side of the PCB.

The sensor was designed with four layers $85 \mathrm{~mm} \times$ $85 \mathrm{~mm} \times 2.4 \mathrm{~mm}$ FR-4 substrate, with $35 \mu \mathrm{m}$ copper thickness. There are 40 interdigitated fingers of copper tracks, with a length $L=75 \mathrm{~mm}$, width $W=2 \mathrm{~mm}$, and space between fingers $S=2 \mathrm{~mm}$. The $W / S$ relationship was chosen based on the conclusions presented in [15], who found that using $W=S$ maximizes the value of capacitance per unit area. To determine the length $L$, we used $L \gg S$, so that the fingers can be considered infinite $[15,16]$.

In the wet end of a paper machine, the sensor must be moved around so that different portions of the pulp can be measured, and wired systems, with sophisticated instruments connected to the sensor (like an impedance analyser), make this task very complicated. In this work, we present a precise handheld wireless capacitive smart sensor that can be easily moved around the wet end of the paper machine and can be read using a smartphone or tablet which has the following features:

(i) It is the first wireless handheld PCB smart sensor that can be moved around the wet end of a paper machine, without heavy and clumsy instruments and cables attached to the sensor

(ii) It is the first capacitive smart sensor based on PCB technology, with all signal processing circuits included in the sensor body

(iii) The novel time interval technique developed for the direct interface to microcontroller technique allows for a much higher resolution in capacitance measurement than the conventional technique proposed by Reverter et al. [17] without using high-frequency oscillators to implement it

(iv) It is extremely low cost, although the results measured with the sensor do not show an error of more than $0.43 \%$ when compared to sophisticated commercial capacitance meters

Since the sensitivity of this type of fringing field capacitive sensor depends on the distance between the copper fingers and the material being measured, we used a conventional solder mask (thickness $=200 \mu \mathrm{m}$ ) over the interdigitated capacitor. This protective layer is necessary to avoid short circuits between the fingers caused by the paper pulp and also to eliminate water adsorption by the FR- 4 .

The PCB internal copper layer close to the bottom side of PCB forms a guard plane that provides shielding from the interference of the high-speed digital signals and also from external electric fields. The internal copper layer close to the top side of the PCB is also used as a guard plane, but it is connected to $V_{\mathrm{dd}}$, to minimize the errors that are induced by the power supply [18]. The top side of the PCB contains the SMD components of the direct interface sensor to microcontroller circuit and the Bluetooth module. Instead of using a solder mask on the top side of the PCB, we protected the components and the FR-4 board with a silicone layer, given that the components will operate in an extremely wet environment. When used in an industrial environment, it is recommended to pot the top of the PCB with an epoxy.

It was observed that, for small capacitance measurements, the wiring between the sensor and the microcontroller is very sensitive to electromagnetic interference and also introduces parasitic capacitances. An important feature of the proposed sensor is that the length of the connection of the sensor with the processing circuit is extremely small, using a PCB via between the top side and the bottom interdigitated capacitor, measuring only approximately $2.4 \mathrm{~mm}$ (the thickness of the FR-4 board). The layout of the PCB interdigitated capacitor is presented in Figure 2.

\section{Signal Processing Circuits}

The signal processing circuits can be divided in two blocks: (a) the capacitance measurement circuit that uses a direct interface sensor to microcontroller and (b) the data transmission circuit, which uses a conventional Bluetooth module to transmit the measured data to an external device (a smartphone or tablet).

4.1. Direct Interface Sensor to Microcontroller. The direct interface sensor to microcontroller technique is an ingenious technique presented by Reverter et al. [17, 19], used to measure resistors and capacitors using only a microcontroller (which contains an internal comparator), without any additional analogue circuits. In this work, we present a novel simple and robust direct interface sensor to microcontroller technique, based on the technique developed by Reverter et al. to measure the sensor's capacitance that allows an increased capacitance measurement resolution without using high-frequency oscillators.

To measure capacitance using the direct interface sensor to microcontroller technique, a system based on an RC circuit is used. In this method, the microcontroller measures the time needed to charge and discharge a capacitor $C_{X}$ between two fixed voltages, through a resistance $R_{X}$. The circuit can be used to measure both $R_{X}$ or $C_{X}$, depending on which one is known. There is another method, which is also regarded as a direct interface sensor to microcontroller, where the microcontroller measures the number of given cycles required to transfer the charge from a reference capacitor to the capacitor under measurement [20-23]. However, this technique is more complex, since it requires an external switched capacitor circuit, and we will not use it in this work. Another interesting technique of charge transfer method to measure capacitance directly with a microcontroller is the QTouch Peripheral Touch Controller, developed by ATMEL [24], where a special processing circuit inside the microcontroller transfers the charge from the measured external capacitor to an internal capacitor in the microcontroller. However, this technique is not well suited for detecting small capacitance changes [25].

A schematic diagram of a simple direct interface sensor to microcontroller circuit used to measure capacitance is shown in Figure 3. 


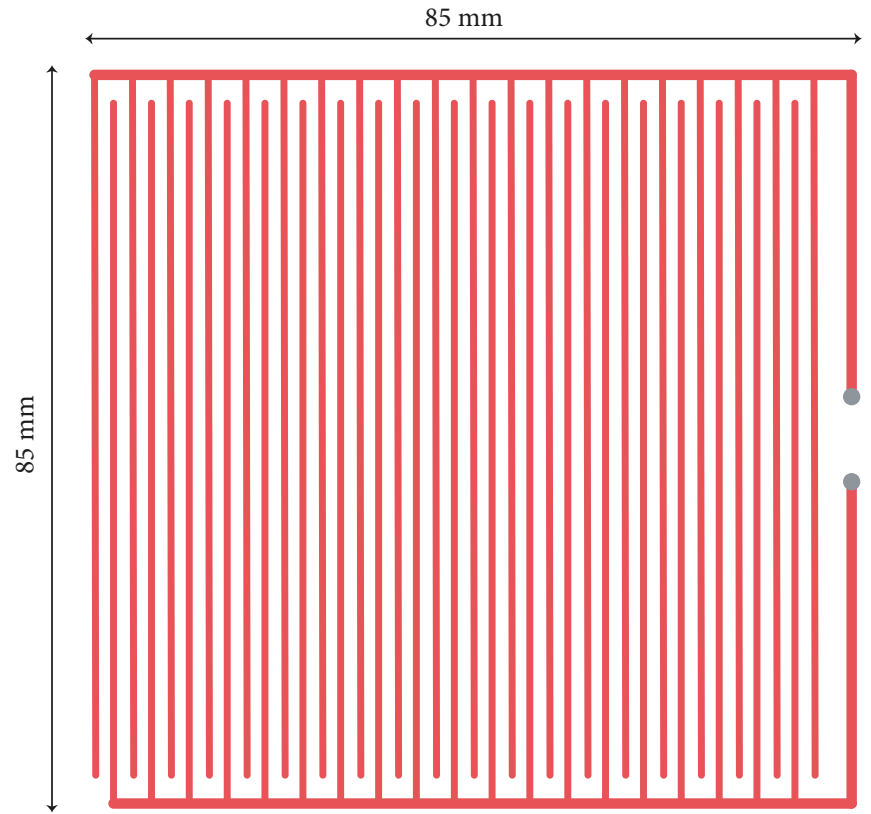

Figure 2: Layout of the PCB interdigitated capacitor with 40 fingers of copper tracks, each finger with a length $L=75 \mathrm{~mm}$, width $W=2 \mathrm{~mm}$, and space between fingers $S=2 \mathrm{~mm}$.

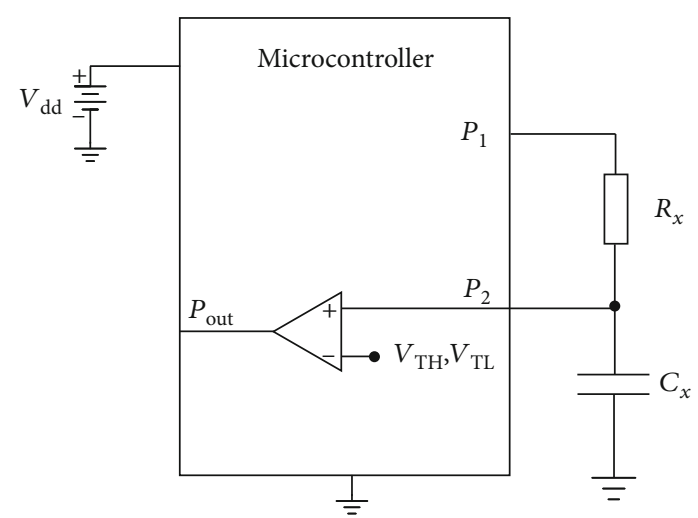

FIgURE 3: Schematic diagram of a direct interface sensor to microcontroller circuit used to measure capacitance. $P_{1}, P_{2}$ and $P_{\text {out }}$ are microcontroller I/O pins.

The circuit operates based on a time-interval technique where during the period of time proportional to the device under measurement, pulses from a constant frequency oscillator are counted. The circuit of Figure 1 is intended to measure the capacitor $C_{X}$ (or resistor $R_{X}$ ); the time interval is given by the phases of charge (or discharge) of the capacitor $C_{X}$ through $R_{X}$. These phases are shown in Figure 4 .

The number of pulses from the oscillator can be counted during the phases of charge $\left(t_{0}-t_{1}\right.$, with $n_{1}$ pulses) or discharge $\left(t_{1}-t_{2}\right.$, with $n_{2}$ pulses). The charge phase is initiated by setting the I/O microcontroller pin $P_{1}$ to logic level " 1 ," putting a voltage equal to the circuit supply voltage $V_{\mathrm{dd}}$ in this pin and charging the capacitor through $R_{X}$.

The voltage at the capacitor $\left(V_{\mathrm{CX}}\right)$ is read by the I/O microcontroller pin $P_{2}$ that is connected to a microcontroller internal comparator with hysteresis, with trip points $V_{\mathrm{TL}}$ and
$V_{\mathrm{TH}}$. The time required to charge a capacitor $C_{X}$ from a voltage $V_{\mathrm{TL}}$ to a voltage $V_{\mathrm{TH}}$ is given by

$$
t_{c}=C_{X} R_{X}\left[\ln \left(\frac{V_{\mathrm{dd}}-V_{\mathrm{TL}}}{V_{\mathrm{dd}}-V_{\mathrm{TH}}}\right)\right] .
$$

The time required to discharge $C_{X}$ from $V_{\mathrm{TH}}$ to $V_{\mathrm{TL}}$ is written as

$$
t_{d}=C_{X} R_{X}\left[\ln \left(\frac{V_{\mathrm{TH}}}{V_{\mathrm{TL}}}\right)\right] .
$$

The terms inside brackets in Equations (2) and (3) are constant, showing that both $t_{c}$ and $t_{d}$ are proportional to $C_{X} R_{X}$. Thus, counting the oscillator pulses during any of these periods of time $t_{d}$ or $t_{c}$, one can easily calculate the value of $C_{X}$ or $R_{X}$, if one of them is known. Due to the smaller noise in the $V_{\mathrm{TL}}$ voltage [18], the oscillator pulses are usually counted during the discharge time $\left(t_{d}\right)$.

\section{Limitations of the Conventional Measurement Technique}

It is important to notice that, for small values of capacitance, the time intervals $t_{c}$ and $t_{d}$ can be very small. For example, if a capacitance $C_{X}=10 \mathrm{pF}$ is being measured, with typical values of $R_{X}=2.2 \mathrm{M} \Omega, V_{\mathrm{dd}}=3.6 \mathrm{~V}, V_{\mathrm{TH}}=1.8 \mathrm{~V}$, and $V_{\mathrm{TL}}=$ $0.9 \mathrm{~V}$, we obtain $\Delta t_{d}=15.3 \mu \mathrm{s}$.

Thus, to be able to detect a $1 \%$ variation in $C_{X}\left(\Delta C_{X}=\right.$ $0.1 \mathrm{pF}$ ), we would have to be able to detect a variation $\Delta t_{d} \approx 153 \mathrm{~ns}$. In such a system, to detect this $\Delta C_{X}=0.1 \mathrm{pF}$ with a difference of only ten counts in the oscillator frequency (what would seem extremely unreliable), we would need a high oscillator frequency of $f_{c k}>50 \mathrm{MHz}$. Since the 


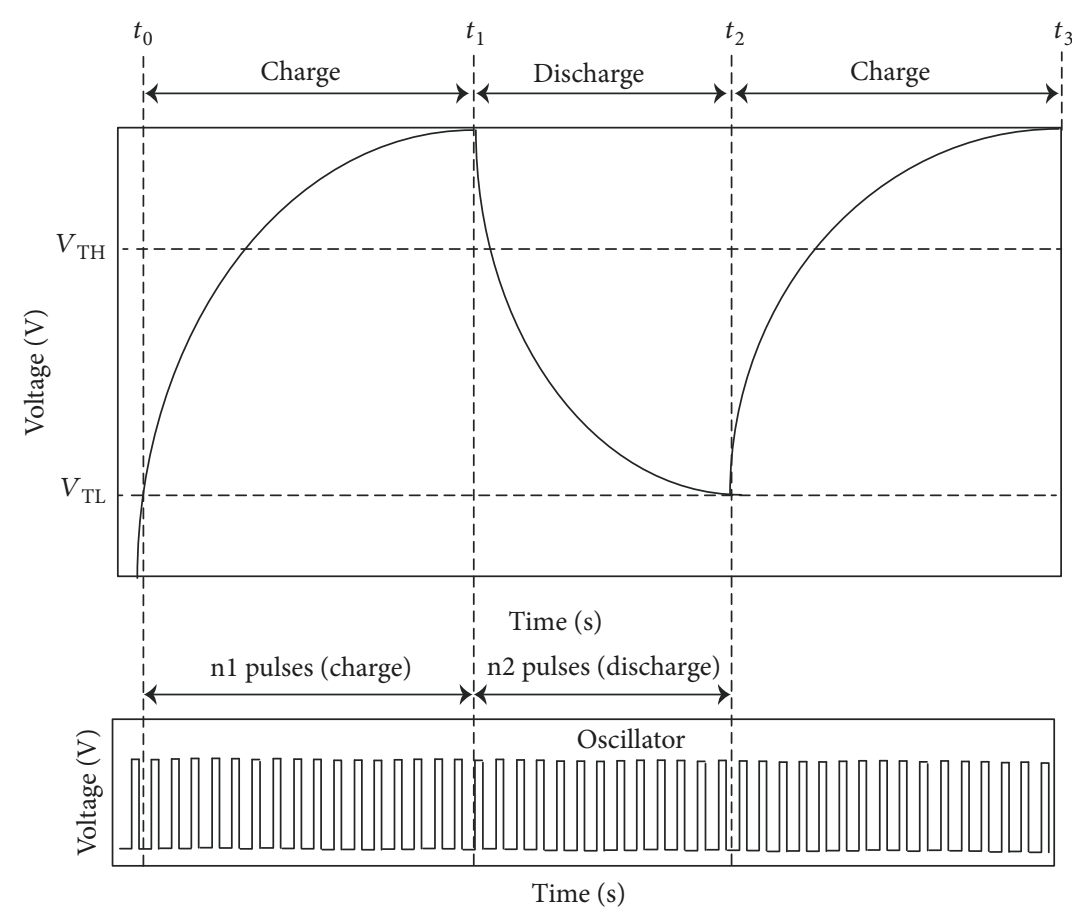

Figure 4: Conventional time-interval technique diagram, showing oscillator pulses and charge/discharge of a capacitor being measured.

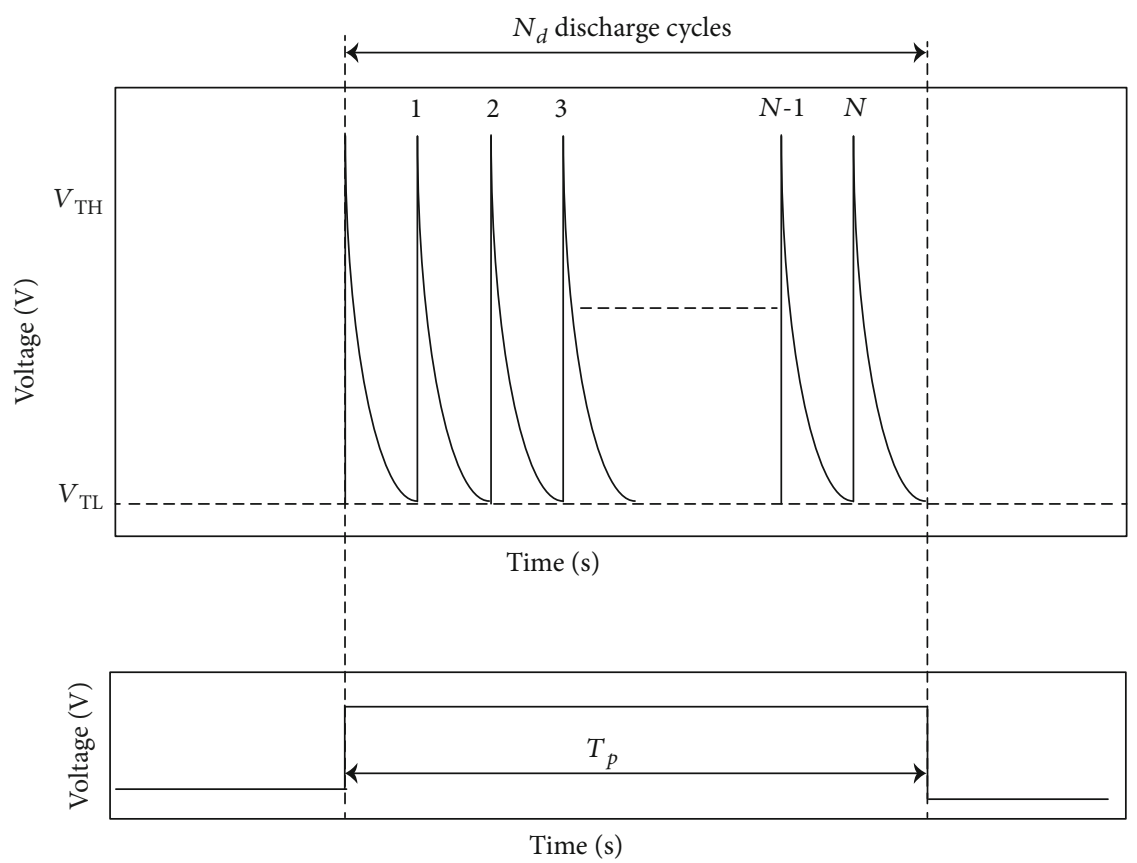

FIgURE 5: Time diagram of the proposed direct interface sensor to microcontroller circuit. $P_{0}, P_{1}, P_{2}$, and $P_{3}$ are microcontroller I/O pins.

value of resistor $R_{0}$ is near the highest practical limit, the conventional time interval technique is very difficult to be used using the microcontroller oscillator.

\subsection{A Novel Direct Interface Sensor to Microcontroller Circuit} Measurement Technique. To allow for the measurement of very small capacitance variations, we developed a different method for reading the direct interface sensor to microcon- troller circuit. Instead of counting pulses from the oscillator, the developed method charges the capacitor almost instantaneously (typically less than $1 \mathrm{~ns}$ ), and then counts the number of discharge cycles, during a fixed time period $T_{p}$. A time diagram of the proposed scheme is presented in Figure 5. If the capacitance measurement does not have to be updated at a fast rate, this period of time $T_{p}$ can be very long (hundreds of milliseconds or even seconds). 


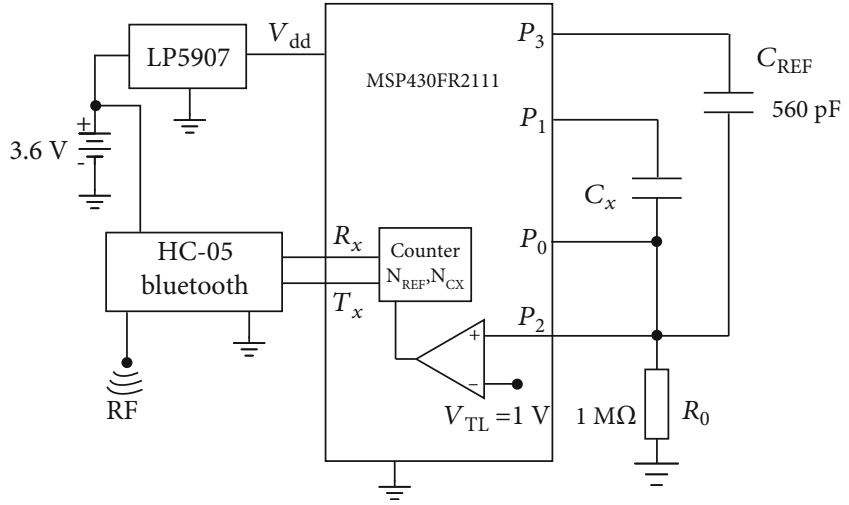

FIGURE 6: Block diagram of the smart sensor circuit.

Using this new scheme with $T_{P}=1 \mathrm{~s}$, the number of discharge cycles for $C_{X}=10 \mathrm{pF}$ is $N_{d 0}=65359$. If $C_{X}$ presents a variation $\Delta C_{x}=0.1 \mathrm{pF}$, the discharge time will be $\Delta t_{d}=$ $15.3 \mu \mathrm{s}+153 \mathrm{~ns}$, and the number of discharge cycles during $T_{P}=1 \mathrm{~s}$ will be $N_{d 1}=64712$, so the difference in the number of counts will be $\Delta N_{d}=N_{d 0}-N_{d 1}=647$, making it easy to detect this small variation of capacitance. Furthermore, since we are taking the average of thousands of measurements, the noise present at the comparator's trip point is filtered.

\section{Circuit Implementation}

To make the sensor portable and taking into account that the current consumption of the signal processing circuit is very low (approximately $50 \mu \mathrm{A}$, including one Bluetooth transmission every $2 \mathrm{~s}$ ), we decided to power the circuit using a single 1.2 A/3.6 V lithium/thionyl chloride battery.

The signal processing circuit used in the smart sensor is presented in Figure 6. Since in this circuit the capacitors are charged to $V_{\mathrm{dd}}$, it is necessary to provide a low-noise and temperature-stable voltage for $V_{\mathrm{dd}}$. So, to provide a stable voltage for $V_{\mathrm{dd}}$, we used the LP5907 from Texas Instruments, an ultralow noise $\left(<6.5 \mu \mathrm{V}_{\mathrm{RMS}}\right) 3.3 \mathrm{~V}$ low drop-out (LDO) voltage regulator, with a typical temperature coefficient of $40 \mathrm{ppm} /{ }^{\circ} \mathrm{C}$ in the $25^{\circ} \mathrm{C}-50^{\circ} \mathrm{C}$ range.

The internal comparator of microcontroller (MSP430FR2111, from Texas Instruments) used for the smart sensor does not depend on $V_{\mathrm{dd}}$. Instead of a voltage given by a fraction of $V_{\mathrm{dd}}$, the comparator's trip point can be programmed (using and internal DAC) to a few fixed values, and in our design, we used the value of $V_{\mathrm{TL}}=1 \mathrm{~V}$.

We can use a very precise and low TC resistor $R_{0}$ (for example, a $0.001 \%$ and $\pm 5 \mathrm{ppm} /{ }^{\circ} \mathrm{C}$ resistor) and eliminate the errors due to this component. However, there are some errors that cannot be cancelled for

(a) The temperature drift in $V_{\mathrm{TL}}$ (approximately $1 \mu \mathrm{V} /{ }^{\circ} \mathrm{C}$ ) defined by $V_{\text {ref }}$, the internal microcontroller's reference voltage

(b) The nonzero output impedance of the CMOS transistors introduce a small series resistance to $R_{0}$ during the discharge cycles (c) Parasitic capacitances in the microcontroller pins influence the value of the capacitance being measured

To eliminate these errors, a self-calibrating circuit is used, where a dummy circuit, with the same configuration of the circuit used to measure the capacitance measures a wellknown capacitor $C_{\mathrm{REF}}$ [26]. The measurement procedure starts with the dummy calibration circuit firstly activated, and the number of discharge cycles with $C_{\mathrm{REF}}$ is obtained. Next, the circuit with the capacitor under measurement $C_{X}$ is activated, the measured number of discharge cycles is compared to the calibration capacitor.

Assuming that both capacitors $\left(C_{\mathrm{REF}}\right.$ and $\left.C_{X}\right)$ are discharged, the measurement starts with the microcontroller's I/O pin $P_{3}$ at "0" logic, $P_{1}$ at high impedance (HZ), and $P_{0}$ set to "1." This forces a voltage equal to $V_{\mathrm{dd}}$ in node $A$ between $C_{\mathrm{REF}}$ and $R_{0}$. Since $P_{3}$ is left in HZ, capacitor $C_{X}$ is floating during this phase.

During the charge cycle, capacitor $C_{\mathrm{REF}}$ is connected directly to the microcontroller pin $P_{0}$ and is charged up to $V_{\mathrm{dd}}$ at a very fast $d V / d T$, limited only by the output current of the microcontroller pin. Since pin $P_{1}$, connected to $C_{X}$, is initially in high impedance (HZ), all current that leaves the microcontroller pin $P_{0}$ goes into $C_{\mathrm{REF}}$. Thus, if the microcontroller's output current limit has a typical value of $8 \mathrm{~mA}$, the time required to charge a $100 \mathrm{pF}$ capacitor would be only $8 \mathrm{~ns}$ and can be neglected compared to the discharge times that are in the order of tens of $\mu$ s.

After $C_{\mathrm{REF}}$ has been totally charged (in the next instruction of the microcontroller, which occurs at least $125 \mathrm{~ns}$ after $P_{0}$ is set to high), $P_{0}$ is set to high impedance, and the capacitor $C_{\mathrm{REF}}$ discharges through $R_{0}$. The comparator detects when the voltage in $C_{\mathrm{REF}}$ reaches $V_{\mathrm{TL}}$, and this discharge time is given by

$$
t_{d \mathrm{REF}}=-R_{0} C_{\mathrm{REF}}\left[\ln \left(\frac{V_{\mathrm{TL}}}{V_{\mathrm{dd}}}\right)\right] .
$$

Next, the same procedure is performed, but using the capacitor under measurement $C_{X}$, and its discharge time is

$$
t_{d \mathrm{CX}}=-R_{0} C_{x}\left[\ln \left(\frac{V_{\mathrm{TL}}}{V_{\mathrm{dd}}}\right)\right] .
$$

The ratio of the discharge time during the calibration phase $\left(t_{d \mathrm{REF}}\right)$ and during the measurement phase $\left(t_{d \mathrm{CXs}}\right)$ is

$$
\frac{t_{d \mathrm{REF}}}{t_{d \mathrm{CX}}}=\frac{C_{\mathrm{REF}}}{C_{X}},
$$

and the value of $C_{X}$ can be easily calculated as

$$
C_{X}=C_{\mathrm{REF}}\left(\frac{t_{d \mathrm{CX}}}{t_{d \mathrm{REF}}}\right) .
$$




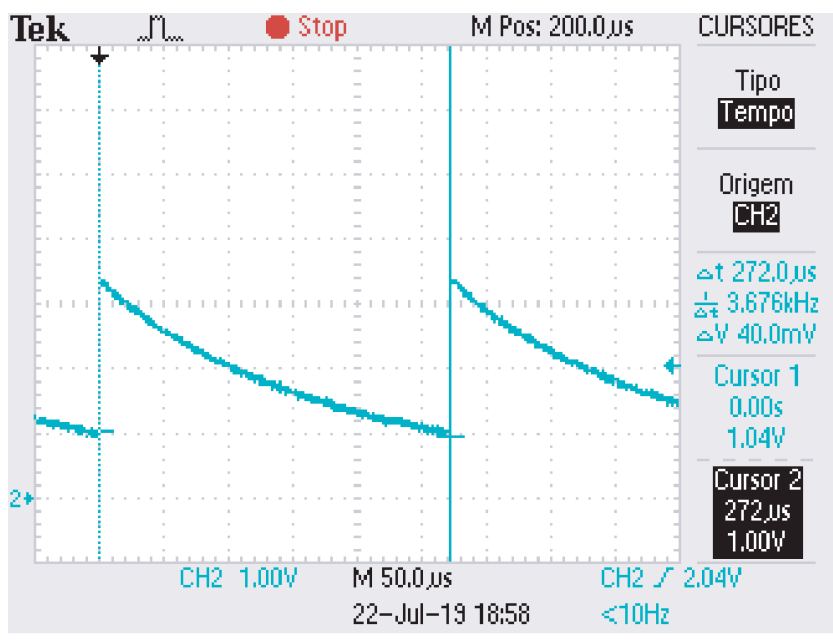

(a) $C_{X}$

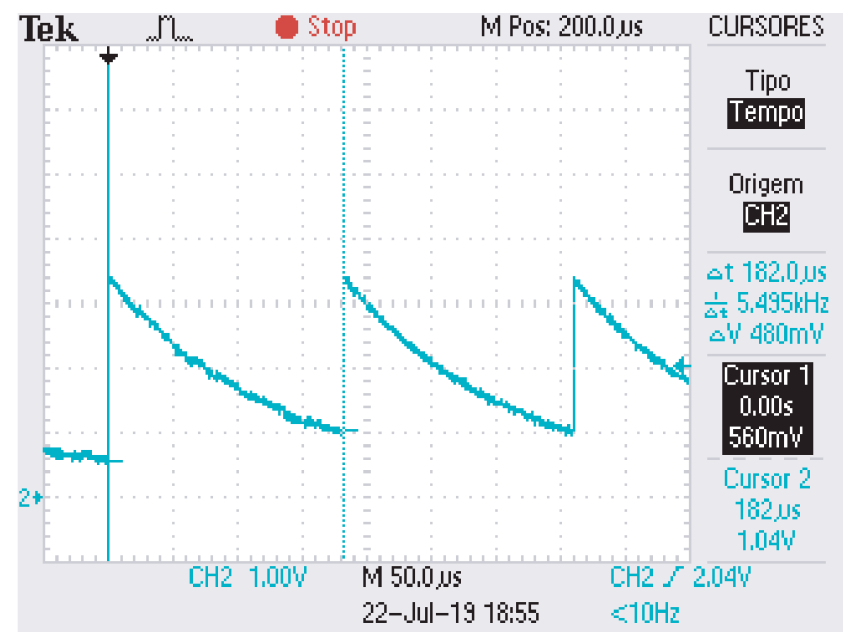

(b) $C_{\mathrm{REF}}$

FIGURE 7: Oscilloscope screens showing the cycles of charge/discharge of the (a) reference capacitor $C_{\mathrm{REF}}$ and (b) sensor's capacitance (in air) $C_{X}$.

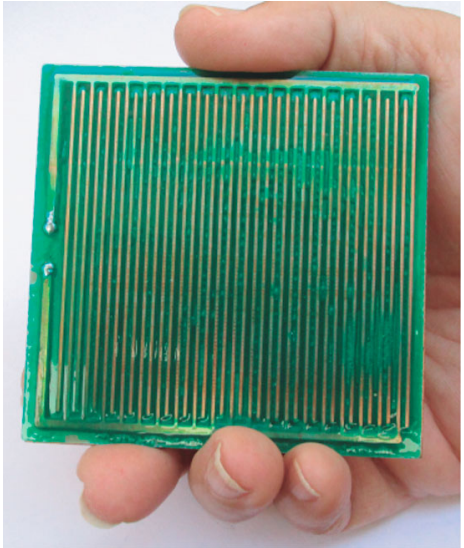

(a)

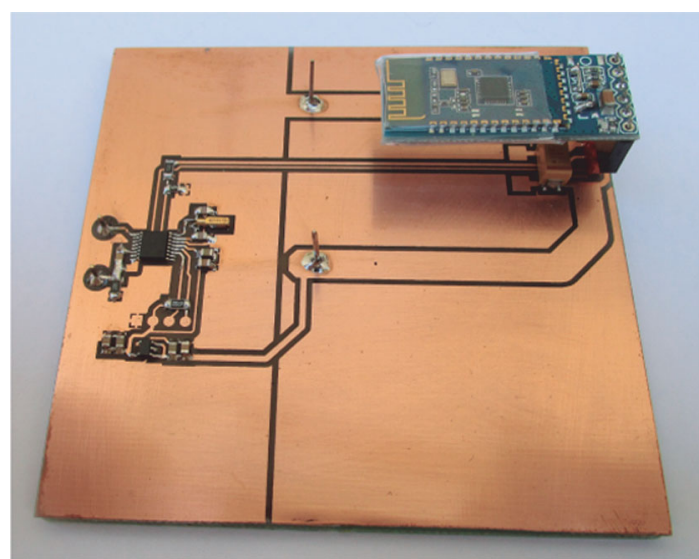

(b)

Figure 8: Photograph of the smart sensor's PCB: (a) bottom side and (b) top side.

The number of pulses counted in each measurement phase is given by

$$
\begin{gathered}
N\left(C_{X}\right)=\frac{T_{P}}{t_{d C \mathrm{X}}}, \\
N\left(C_{\mathrm{REF}}\right)=\frac{T_{P}}{t_{d C \mathrm{REF}}} .
\end{gathered}
$$

Therefore, combining Equations (7), (8), and (9), we conclude that

$$
C_{X}=C_{\mathrm{REF}}\left(\frac{N_{\mathrm{REF}}}{N_{\mathrm{CX}}}\right)
$$

and we have a linear and easy to use relationship to calculate $C_{X}$ from the values of $N_{\mathrm{CX}}$ and $N_{\mathrm{REF}}$.

A print of the oscilloscope screens showing the instantaneous charge and the discharge time (which depends on the capacitor value) of both the reference capacitor $C_{\mathrm{REF}}$ and the sensor's capacitance (in air) $C_{X}$ is shown if Figure 7.

To obtain a precise measurement periods $T_{p}$ (we used $\left.T_{p}=1 \mathrm{~s}\right)$, a real time clock crystal $(f=32768 \mathrm{~Hz})$ was used. The timer which defines $T_{p}$ starts with the transition of $P_{0}$ from $\mathrm{HZ}$ to high. After the measuring period $T_{p}$ ends, the next transition of $P_{0}$ from $\mathrm{HZ}$ to high starts the timer $T_{p}$ again, to measure the unknown capacitor $C_{x}$.

In this prototype, where we want to test if it is possible to fabricate high-speed digital and communication circuits in the top layer of the PCB sensor without interfering with the capacitive measurement, a commercial HC-05 Bluetooth module was soldered on the top of the smart sensor PCB. This module transmits the measured data to an external device (a smartphone or tablet). Of course, in a final product, a Bluetooth module like the DA14531-00000OG2 from Dia$\log$ Semiconductors should be used, which would make it easy to epoxy the PCB top side.

It is important to notice that the communication protocol between the microcontroller and the Bluetooth module is 


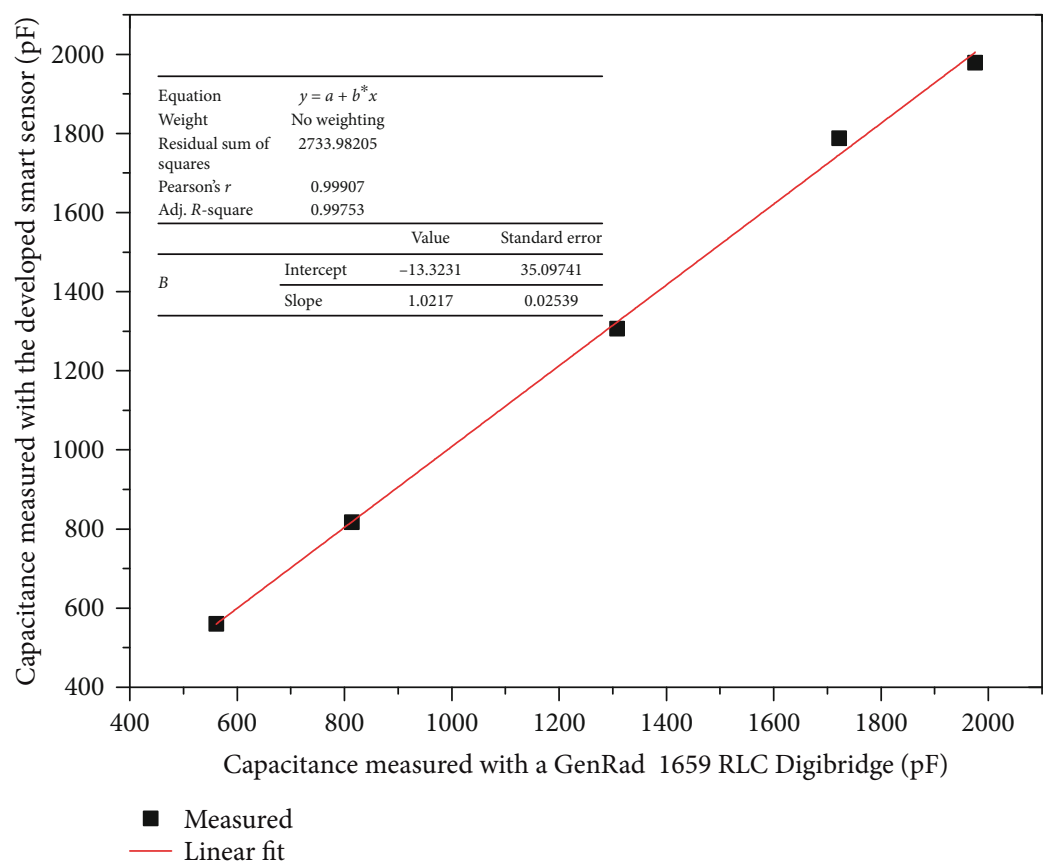

FIGURE 9: Sensor's calibration curve.

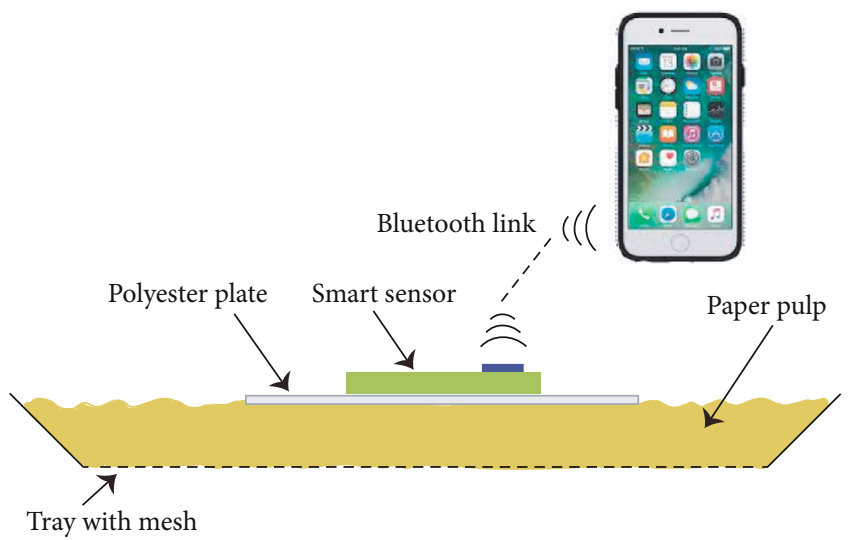

FIgURE 10: Schematic diagram of the measurement set-up.

exactly the same if we use an HC-05 or a low-cost Bluetooth module like the DA14531-00000OG2, so nothing would need to be changed. A photograph of the smart sensor's PCB, without the battery connected, is shown in Figure 8.

\section{Experimental Results}

7.1. Sensor's Calibration. A circuit of the proposed capacitance measurement circuit was reproduced and implemented on the top of a two-layer PCB, without the interdigitated capacitor on the bottom side. Commercial capacitors were firstly measured with a GenRad 1659 RLC Digibridge, and then measured using our direct to microcontroller interface circuit.

In Figure 9, we show a plot of the measured capacitance values with our sensor, as a function of the values obtained with the GenRad 1659 RLC Digibridge. The maximum calcu-

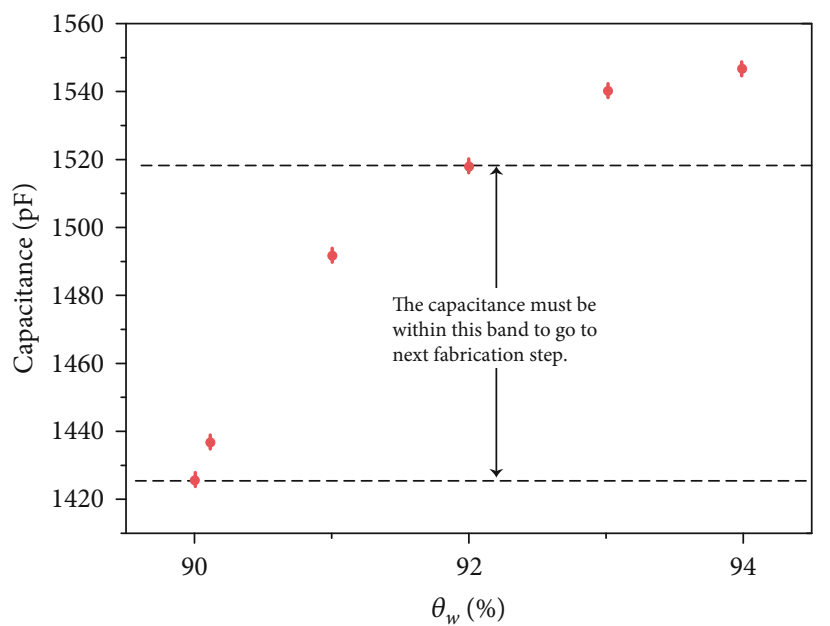

FIgURE 11: Measured capacitance (average from five measurements), as a function of the gravimetric water content in a paper pulp.

lated difference between the measurements was only $0.43 \%$, for a very large capacitance range, from $560 \mathrm{pF}$ to $1.98 \mathrm{nF}$.

7.2. Materials and Methods. The paper pulp at the end of a paper machine is basically a suspension composed of water and paper fibres. This suspension is spread over a mesh tray (known in the paper industry as the "wire"), and the moisture content of this paper pulp has to be measured, in order to allow it continues to the next fabrication step.

To prepare a set-up measurement for our sensor, we built a small mesh (a circular tray with a diameter of $30 \mathrm{~cm}$ ) to emulate, in a laboratory, the conditions found in the wet end of an industrial single-side paper fabrication machine. 


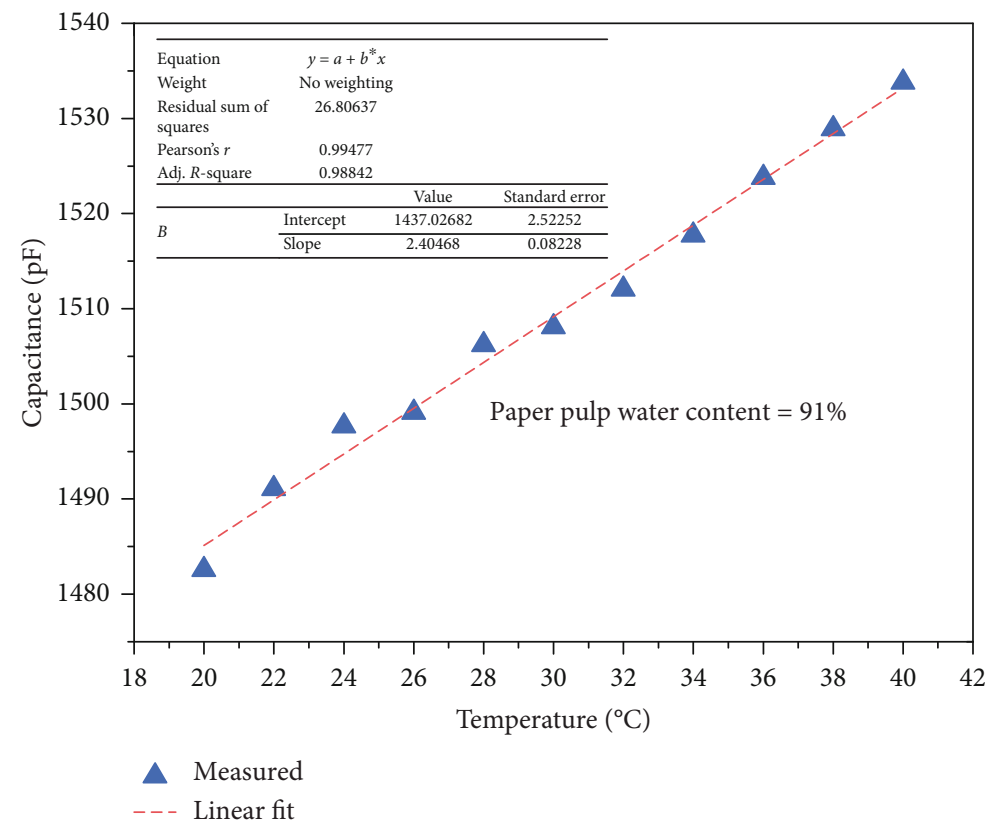

FIGURE 12: Variation of capacitance as a function of the temperature.

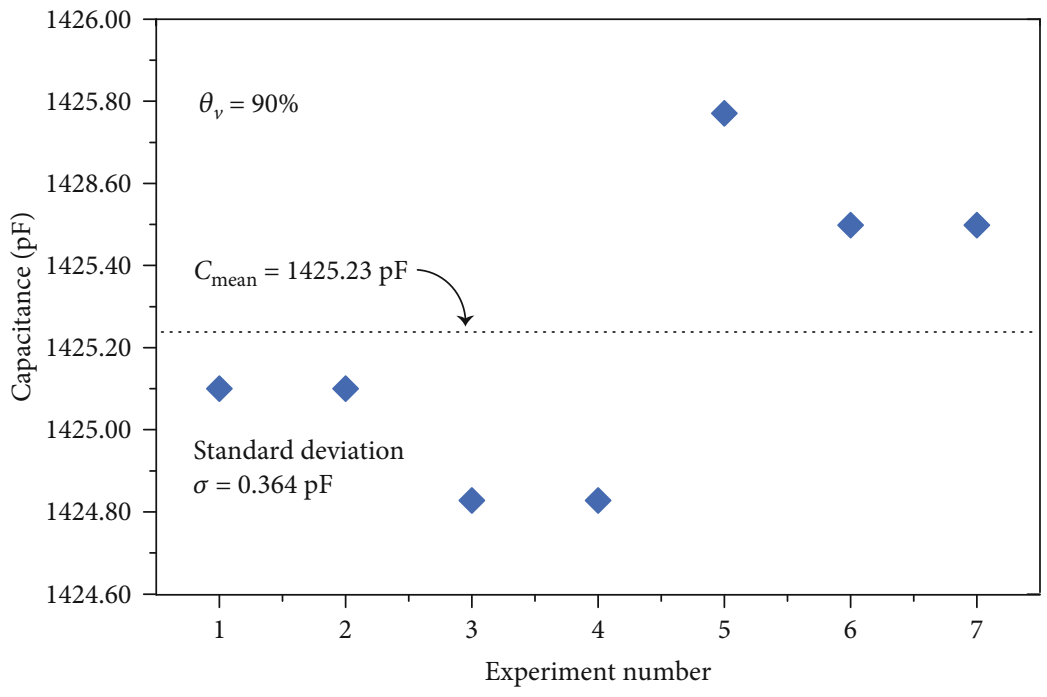

FIgURE 13: Repeatability test for a paper pulp with $\theta_{w}=90 \%$.

The sensor was installed on a thin transparent polyester plate (with a thickness of $0.5 \mathrm{~mm}$ ), so that the apparatus can be placed on the paper pulp, ensuring a large surface with good contact between the paper pulp and the polyester plate.

In our experiment, the paper pulp suspension was obtained by liquidizing (using a conventional kitchen's blender) controlled amounts of a tissue paper and water, weighted in a precision scale. In Figure 10, we show a schematic diagram of the measurement set-up.

Firstly, we prepared six suspensions of paper pulp with gravimetric water contents in the range $\theta_{w}=90 \%$ to $\theta_{w}=$ $94 \%$, each sample with a volume of approximately one liter. The smart sensor was programmed to transmit continuously the values of $N_{\mathrm{REF}}$ and $N_{\mathrm{CX}}$, so that every $2 \mathrm{~s}$ we can calculate the value of $C_{X}$, and the values of $N_{\mathrm{REF}}$ and $N_{\mathrm{CX}}$ were read in a smartphone. For each value of paper pulp with different values of $\theta_{w}$, we took several measurements, without moving the sensor on the paper pulp surface.

In Figure 11, we present a plot of the measured capacitance (the average from five measurements, taken every two seconds), as a function of the volumetric water content in the paper pulp, at $T=25 \mathrm{C}$. As it can be observed, the variation is nonlinear, as with other PCB-based sensors used to measure water content.

This is easily explained by the fact that a PCB capacitive sensor has a fixed value capacitor (defined by the dielectric composed of the solder mask film) in parallel with one varying capacitor, which is defined by the dielectric composed of the paper pulp. The resulting capacitance of this parallel association is nonlinear [10]. 


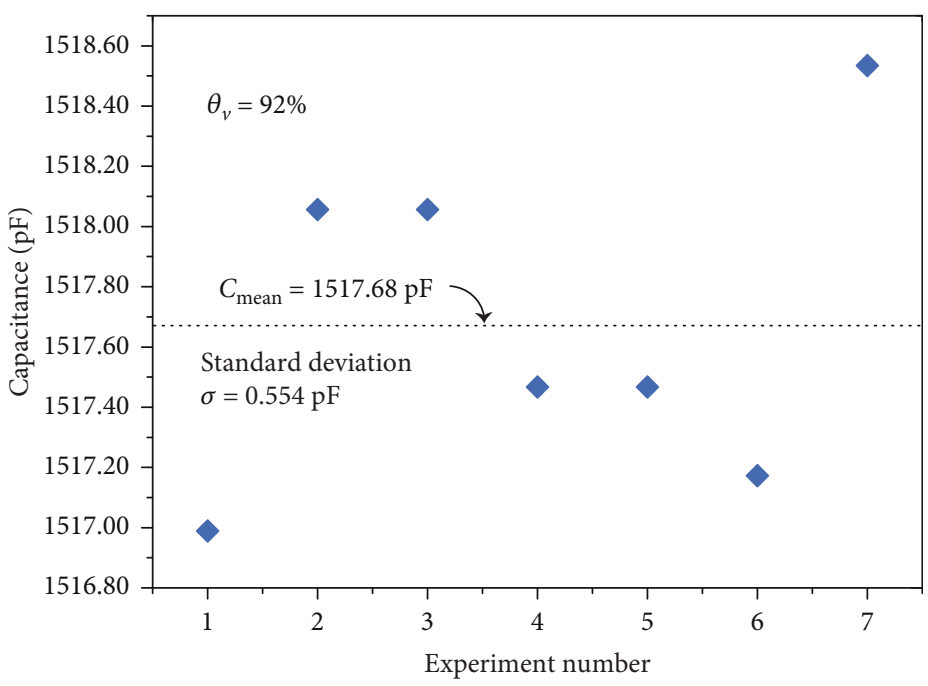

FIGURE 14: Repeatability test for a paper pulp with $\theta_{w}=92 \%$.

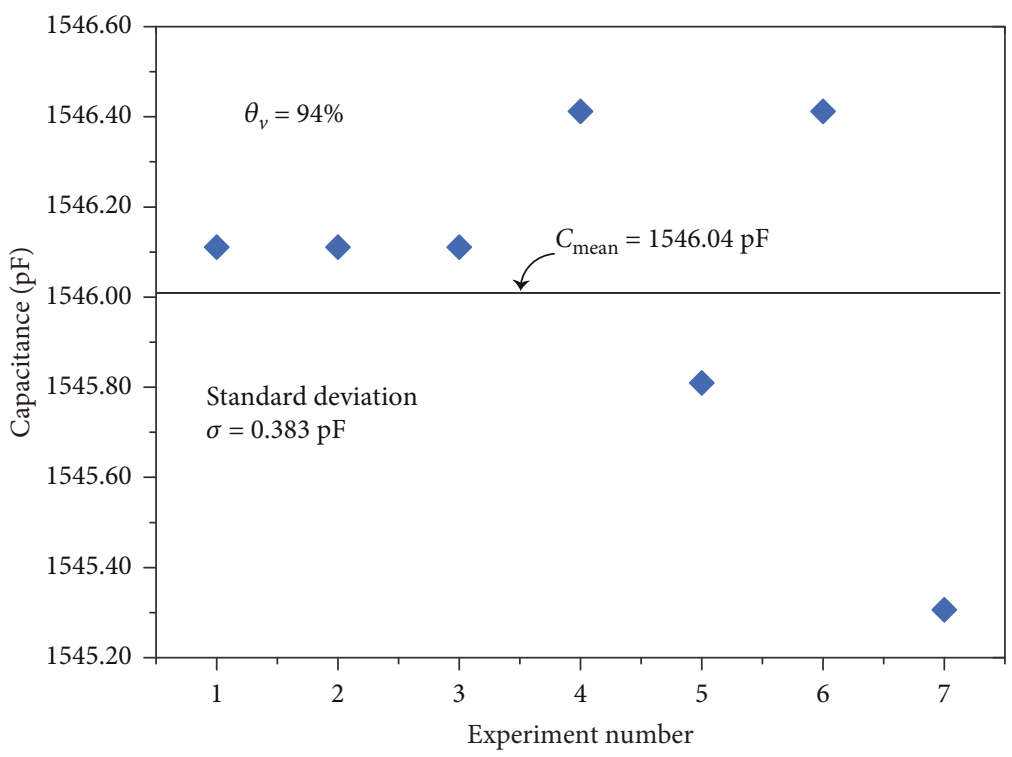

Figure 15: Repeatability test for a paper pulp with $\theta_{w}=94 \%$.

7.3. Sensor's Variation with Temperature. It has been shown in [27] that fringing field capacitive sensors are sensitive to temperature variations. Since the operator at the wet end of the paper machine can easily obtain the temperature of the paper pulp, it is important to provide a temperature correction to the capacitance measured in the paper pulp.

To obtain the correction equation, a simple experiment was conducted. A tray with paper pulp with $91 \%$ of water content was prepared, and the developed sensor was used to measure the paper pulp. The system was inserted into a thermal chamber and submitted to temperature variations in the range of $20^{\circ} \mathrm{C}$ to $40^{\circ} \mathrm{C}$. It is assumed that the water content does not change during the measurement period, and the measured change in capacitance is only due to the sensor's temperature sensitivity. The measured results are plotted in Figure 12.
TABLE 1: Calculated values of the mean and the standard deviation in the repeatability tests.

\begin{tabular}{lcc}
\hline$\theta_{v}$ & Mean & Standard deviation \\
\hline $90 \%$ & $1425.24 \mathrm{pF}$ & $0.364 \mathrm{pF}$ \\
$92 \%$ & $1517.68 \mathrm{pF}$ & $0.554 \mathrm{pF}$ \\
$94 \%$ & $1546.04 \mathrm{pF}$ & $0.383 \mathrm{pF}$ \\
\hline
\end{tabular}

As one can observe, the change in capacitance is practically linear with the temperature, so we can write

$$
C(T)=C_{0}\left[1+\alpha\left(T-T_{0}\right)\right]
$$

where $C_{0}$ is the value of the capacitance measured at $25^{\circ} \mathrm{C}$ (as shown in Figure 11), $T_{0}=25 \mathrm{C}, T$ is the temperature 


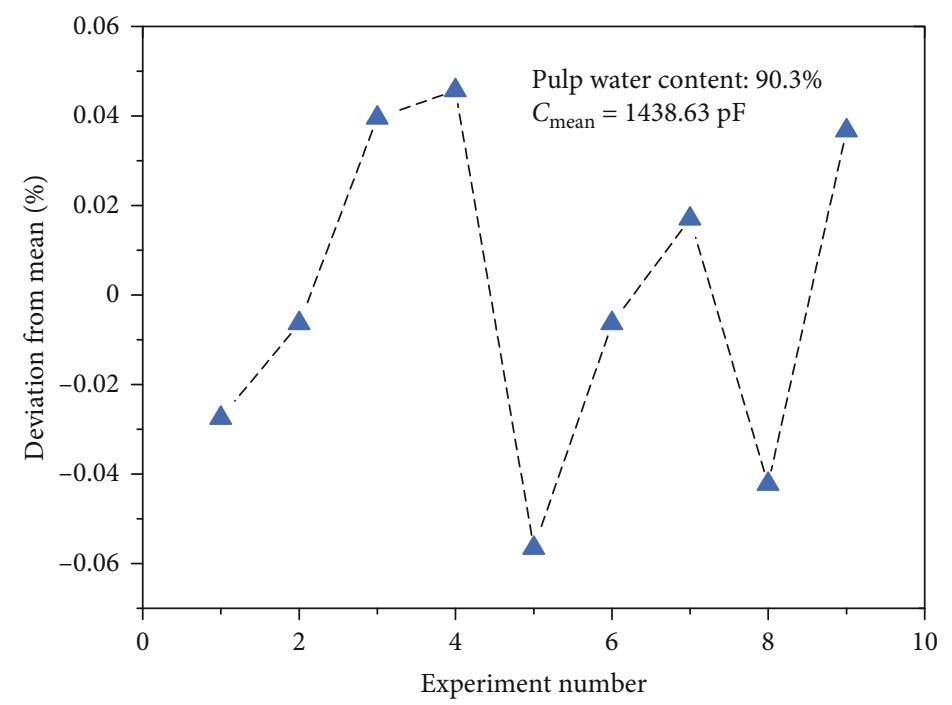

FIGURE 16: Measured capacitance and calculated deviation from the mean (in \%) for the reproducibility test.

measured in the paper pulp, $C(T)$ is the measured value of the capacitance, and $\alpha$ is the value of $d C / d T$, calculated as $\alpha=2.4 \mathrm{pF} /{ }^{\circ} \mathrm{C}$ from the slope of the linear equation presented in Figure 12.

Thus, after measuring $C(T)$ and $T$, the value of $C_{0}$ can be easily calculated using Equation (2). With $C_{0}$ calculated, we can determine if it is within the desired capacitance range of $1428 \mathrm{pF}$ to $1519 \mathrm{pF}$, corresponding to the water content range $(60 \%$ to $92 \%)$. This can be easily implemented in the smartphone software.

\section{Repeatability and Reproducibility Test}

During the paper fabrication process, the repeatability of the water content measurements in the paper pulp is important to obtain a constant paper quality. Thus, we ran a series of tests to evaluate the developed smart sensor in what concerns repeatability and reproducibility.

8.1. Repeatability Tests. We performed repeatability tests for paper pulp with a water content $\theta_{w}=90 \%, \theta_{w}=92 \%$, and $\theta_{w}=94 \%$. The emulsion was placed on the mesh and the sensor was installed. Next, without disturbing the paper pulp or the sensor, we took seven capacitance measurements, in intervals of $2 \mathrm{~s}$. It is assumed that the paper pulp cannot change its moisture content during this small period of time [9]. In Figures 13-15, we present the results of these tests. The calculated values of the mean and the standard deviation are shown in Table 1. It is observed that the worst case occurred for $\theta_{v}=92 \%$, when the calculated standard deviation is $\sigma=554 \mathrm{fF}$, four orders of magnitude lesser than the mean value $C_{\text {mean }}=1517.68 \mathrm{pF}$.

8.2. Reproducibility Test. To emulate a fabrication environment, where the operator moves the sensor around the wire region to check the paper pulp moisture, we conducted a reproducibility test. The reproducibility test evaluates the ability of the sensor to be installed and removed several times, reading the same paper pulp with consistent values.

In our test, we prepared a paper pulp, installed the sensor, and made one measurement. Next, the sensor was removed, cleaned, and a new measurement was taken with the same paper pulp. This was repeated nine times, and the results are shown in Figure 16. The maximum deviation from the mean value was only $0.006 \%$, showing that the sensor can be moved around the wet end of the machine and used to measure different portions of the paper pulp with consistent results. A sample of paper pulp with $\theta_{w}=90.3 \%$ was used in this reproducibility test.

\section{Conclusions}

A capacitive fringing field smart sensor designed to measure the water content in the paper pulp in the wet end of a paper machine was developed and tested in laboratory. The interdigitated capacitive sensor is based on standard PCB technology. The smart sensor was fabricated using a four-layer PCB, which allows for adding a ground shield plane and a $V_{\text {dd }}$ plane in the inner layers of the PCB. The interdigitated capacitor is patterned in the bottom side of the PCB and the signal processing circuits are soldered on the PCB's top side.

This construction technique eliminates the need for wiring between the sensor and the signal processing circuits (the connection is extremely short, made with a PCB via between the top and bottom layers), eliminating the stray capacitances and the interference that could be coupled through the wiring. A novel signal processing technique was developed, which allowed for the system to measure very small deviations of capacitance using a low-frequency oscillator.

A circuit of the proposed capacitance measurement circuit was reproduced and implemented on the top of a twolayer PCB, without the interdigitated capacitor on the bottom side. Commercial capacitors were firstly measured with a GenRad 1659 RLC Digibridge, and then measured using 
our direct to microcontroller interface circuit. The maximum calculated difference between the measurements was only $0.43 \%$, for a large capacitance range, from $560 \mathrm{pF}$ to $1.98 \mathrm{nF}$.

The sensor was tested in a laboratory using a set-up which emulated the wet end of a paper machine. Although the paper pulp enters the wet end of a paper machine with a high moisture content (typically 99\%), it is necessary to wait until it reaches the range $\theta_{w}=90 \%$ to $92 \%$ until it can go to the next fabrication step. In this $\theta_{w}$ range, a variation of $\Delta C=92.1 \mathrm{pF}$ was measured. Repeatability tests showed that the measured values presented a deviation from the mean that are four orders of magnitude lesser than the mean. Reproducibility tests, made with nine different measurements in the same paper pulp, showed that the maximum error observed was less than $0.006 \%$.

The smart sensor was tested in the $20^{\circ} \mathrm{C}$ to $40^{\circ} \mathrm{C}$ temperature range, in a paper pulp with a gravimetric water content of $91 \%$. Since the variation of capacitance with temperature is practically linear, we propose a simple linear compensation equation to correct for the observed sensitivity with the temperature.

The measured data is transmitted wireless (via Bluetooth), eliminating connections between the sensor and the paper machine controllers.

\section{Data Availability}

The measured data used to support the findings of this study are included within the article.

\section{Conflicts of Interest}

The authors declare that they have no conflicts of interest.

\section{Acknowledgments}

This study was financed in part by the CAPES-Brazil-Finance Code 001.

\section{References}

[1] S. Nakayama, "Microwave measurements of low pulp concentration in papermaking process," Japanese Journal of Applied Physics, vol. 33, Part 1, 6A, pp. 3614-3616, 1994.

[2] Z. Q. Wu, W. J. Batchelor, and R. E. Johnston, "Development of an impedance method to measure the moisture content of a wet paper web," Appitta Journal, vol. 52, no. 6, pp. 425428, 1999.

[3] S. Okamura and Y. Zhang, "New method for moisture content measurement using phase shifts at two microwave frequencies," Journal of Microwave Power and Electromagnetic Energy, vol. 35, no. 3, pp. 175-178, 2000.

[4] S. Nakayama, "Microwave measurements of moisture content of aggregate," Japanese Journal of Applied Physics, vol. 33, Part 1, 5A, pp. 2809-2810, 1994.

[5] L. Chase and J. D. Goss, "Method and apparatus for measuring and controlling the surface characteristics of sheet materials such as paper," US Patent 5654799, 1997.
[6] J. D. Watson, C. Hagart-Alexander, J. D. Goss, J. G. Preston, $\mathrm{H}$. $\mathrm{Hu}$, and L. Dudas, "Wet end control for papermaking machine," US Patent 6,086,716, 2000.

[7] L. Chase, C. J. Goss, and T. V. Graham, "Electromagnetic field perturbation sensor and methods for measuring water content in sheetmaking systems," US Patent 5954923, 1999.

[8] S. Simula, S. Ikäläinen, K. Niskanen, T. Varpula, H. Seppä, and A. Paukku, "Measurement of the dielectric properties of paper," Journal of Imaging Science and Technology, vol. 43, no. 5, pp. 472-477, 1999.

[9] K. Sundara-Rajan, L. ByrdII, and A. V. Mamishev, "Moisture content estimation in paper pulp using fringing field impedance spectroscopy," IEEE Sensors Journal, vol. 4, no. 3, pp. 378-383, 2004.

[10] E. da Costa, N. de Oliveira, F. Morais et al., "A Self-Powered and autonomous fringing field capacitive sensor integrated into a micro sprinkler spinner to measure soil water content," Sensors, vol. 17, no. 3, p. 575, 2017.

[11] R. N. Dean, J. D. Craven, E. A. Guertal, and K. A. Varnavas, "A PCB Sensor for status monitoring of stored food stocks," IEEE Sensors Letters, vol. 3, no. 4, pp. 1-4, 2019.

[12] R. N. Dean, A. K. Rane, M. E. Baginski, J. Richard, Z. Hartzog, and D. J. Elton, “A capacitive fringing field sensor design for moisture measurement based on printed circuit board technology," IEEE Transactions on Instrumentation and Measurement, vol. 61, no. 4, pp. 1105-1112, 2012.

[13] R. N. Dean, A. Rane, M. Baginski, J. Richard, Z. Hartzog, and D. Elton, "Capacitive fringing field sensors in printed circuit board technology," in 2010 IEEE Instrumentation \& Measurement Technology Conference Proceedings, pp. 970-974, Austin, TX, USA, May 2010.

[14] P.-Y. Chen, C.-C. Chen, W.-K. Yeh et al., "Using capacitance sensor to extract characteristic signals of dozing from skin surface," Journal of Sensors, vol. 2014, Article ID 238350, 7 pages, 2014.

[15] R. Igreja and C. J. Dias, "Analytical evaluation of the interdigital electrodes capacitance for a multi- layered structure," Sensors and Actuators A: Physical, vol. 112, no. 2-3, pp. 291-301, 2004.

[16] R. Igreja and C. J. Dias, "Extension to the analytical model of the interdigital electrodes capacitance for a multi-layered structure," Sensors and Actuators A: Physical, vol. 172, no. 2, pp. 392-399, 2011.

[17] F. Reverter, M. Gasulla, and R. Pallàs-Areny, A Low-Cost Microcontroller Interface for Low-Value Capacitive Sensors, in Proc, IEEE IMTC, Como, Italy, 2004.

[18] F. Reverter, M. Gasulla, and R. Pallàs-Areny, "Analysis of power-supply interference effects on direct sensor-tomicrocontroller interfaces," IEEE Transactions on Instrumentation and Measurement, vol. 56, no. 1, pp. 171-177, 2007.

[19] F. Reverter and R. Pallàs-Areny, Direct Sensor-toMicrocontroller Interface Circuits. Design and Characterization, Marcombo, Barcelona, Spain, 2005.

[20] P. H. Dietz, D. Leigh, and W. S. Yerazunis, "Wireless liquid level sensing for restaurant applications," in SENSORS, 2002 IEEE, pp. 715-719, Orlando, FL, USA, USA, June 2002.

[21] B. George and V. J. Kumar, "Switched capacitor signal conditioning for differential capacitive sensors," IEEE Transactions on Instrumentation and Measurement, vol. 56, no. 3, pp. 913917, 2007.

[22] J. E. Gaitan-Pitre, M. Gasulla, and R. Pallas-Areny, "Analysis of a direct interface circuit for capacitive sensors," IEEE 
Transactions on Instrumentation and Measurement, vol. 58, no. 9, pp. 2931-2937, 2009.

[23] S. Ogawa, Y. Oisugi, K. Mochizuki, and K. Watanabe, "A switched capacitor interface for differential capacitance transducers," IEEE Transactions on Instrumentation and Measurement, vol. 50, no. 5, pp. 1296-1301, 2001.

[24] “Atmel - AT42QT1070 Seven channel QTouch ${ }^{\circledR}$ Touch Sensor IC Datasheet," http://www.atmel.com.

[25] L. Capineri, "Design and realization of a tactile switches module with capacitive sensing method implemented with a microcontroller," Transactions on Environment and Electrical Engineering, vol. 1, no. 3, 2016.

[26] F. Reverter, "The art of directly interfacing sensors to microcontrollers," Journal of Low Power Electronics and Applications, vol. 2, no. 4, pp. 265-281, 2012.

[27] R. B. McIntosh and M. E. Casada, "Fringing field capacitance sensor for measuring the moisture content of agricultural commodities," IEEE Sensors Journal, vol. 8, no. 3, pp. 240$247,2008$. 